\title{
Sarcoma Pleomórfico Indiferenciado. A propósito de un caso
}

\section{Undifferentiated Pleomorphic Sarcoma. About a case}

Oxmany Lantigua Hernández. ${ }^{1}$, Yania González Díaz. ${ }^{2}$, Mario Miguel Morales Wong. ${ }^{3}$ \& Ricardo Alfaro Alonso. ${ }^{4}$

DOI: https://doi.org/10.33262/anatomiadigital.v4i1.1533

\begin{abstract}
.
Resumen.

Introduction: Undifferentiated

Introducción: El sarcoma pleomórfico Pleomorphic sarcoma is a rare and indiferenciado es un cáncer raro y aggressive cancer from as early as 1977 was agresivo. Desde tiempos tan tempranos considered the most frequent soft tissue como en 1977 era considerado el sarcoma sarcoma in adults, although its diagnosis de partes blandas más frecuente en adultos, and evolution continue to be an Enigma. A 77-year-old male patient is described who no obstante, su diagnóstico y evolución continúan siendo un enigma. Se describe

\footnotetext{
${ }^{1}$ Hospital Dr. Mario Muñoz Monroy. Colón, Cuba, ygd75@nauta.cu ID https://orcid.org/0000-0003-1921-9283

${ }^{2}$ Hospital Dr. Mario Muñoz Monroy. Colón, Cuba, Yolanda2148@ nauta.cu iD https://orcid.org/0000-0001-9907-8892

${ }^{3}$ Hospital Dr. Mario Muñoz Monroy. Colón, Cuba, mmorales.mtz@infomed.sld.cu iD https://orcid.org/0000-0001-89012662

${ }^{4}$ Hospital Dr. Mario Muñoz Monroy. Colón, Cuba, ricardoalfaro.mtz@infomed.sld.cu 0015-1840
} 
began 11 months ago with an increase in volume in the left lateral region of the abdomen and attended the consultation because in the last two months he had had an exaggerated growth and bleeding from the surface of the tumor. A trucut biopsy was performed, resulting in a pleomorphic sarcoma of unknown origin. The size, depth and metastasis contra indicated the surgery evolving unfavorably. Methodology: An analysis and synthesis of the biography was carried out, the fourth my uncle made deductions in relation to the production and future development of similar cases. Conclusions: Undifferentiated pleomorphic sarcoma is one of the few sarcomas that can present as a superficial tumor, it is necessary to always take it into account by dermatologists, clinical surgeons and family doctors who are those closest to the patients Because, due to their aggressive behavior, the best prognosis for cure and survival of affected patients is early detection. And therefore, the opportune treatment of choice, surgical, this would be of great benefit to patients

Keywords: undifferentiated pleomorphic sarcoma. Soft tissue. Abdominal wall. malignant fibrous histiocytoma un paciente masculino de 77 años que comienza 11 meses atrás con aumento de volumen en región lateral izquierda de abdomen y acude a consulta porque en los últimos 2 meses ha tenido un crecimiento exagerado y sangrado por la superficie del tumor. Se realiza biopsia por trucut resultando un sarcoma pleomórfico de origen desconocido. El tamaño, la profundidad y la metástasis contraindicaron la cirugía, evolucionando desfavorablemente. Metodología: Se realizó un análisis y síntesis de la bibliografía lo cual permitió hacer deducciones en relación al desarrollo y proyecciones futuras de casos semejantes. Conclusiones: El sarcoma pleomórfico indiferenciado es uno de los pocos sarcomas que se pueden presentar como un tumor superficial, es necesario tenerlo siempre en cuenta por parte de dermatólogos, cirujanos, clínicos y médicos de la familia que son los más cercanos a los pacientes pues por su comportamiento agresivo, el mejor pronóstico de curación y sobrevida de los pacientes afectados es la detección precoz y por consiguiente el tratamiento oportuno; de elección el quirúrgico. Esto redundaría en un gran beneficio para los pacientes.

Palabras claves: Sarcoma pleomórfico indiferenciado. Partes blandas. Pared abdominal. Fibrohistiocitoma maligno

\section{Introducción.}

El sarcoma pleomórfico indiferenciado clasificado anteriormente hasta el 2002 como fibrohistiocitoma maligno (FMH) (Fletcher CDM, Unni KK \& Mertens F. (Eds.), 2002) es un cáncer raro y agresivo que aparece tanto en las partes blandas como en el hueso. Fue 
descrito por vez primera por O'Brien JE \& Stout AP en 1964 y desde ese momento ha sido objeto de controversia. Estos autores describieron el fibrohistiocitoma maligno (FHM) como un tumor rico en histiocitos y fibroblastos con patrón de crecimiento estoriforme. Ya en 1977 según Weiss et al, 2007 el fibrohistiocitoma maligno (FHM) era considerado el sarcoma de partes blandas más frecuente en los adultos; pese a esto continúa siendo un enigma. A lo largo de la última década se han publicado un compendio de evidencias que sugieren que el FHM constituye la ruta final común de distintos tumores que experimentan una evolución progresiva hacia la desdiferenciación. (Martínez-Navarro, 2019; Guerra, Lamadrid \& Rodríguez Machado, 2017).

En tanto continúe sin dilucidarse cómo clasificar de forma exacta estos tumores, el término "fibrohistiocitoma maligno" sigue constituyendo el diagnóstico de miles de pacientes y continúa siendo comúnmente utilizado tanto por los médicos como por los pacientes. (Martínez-Navarro, 2019).

El sarcoma pleomórfico de partes blandas se presenta de forma típica en pacientes de entre 50 y 70 años, aunque puede aparecer a cualquier edad, muy raro en menores de 20 años con un ligero predominio del sexo masculino en una proporción 2:1. La forma de presentación cutánea más frecuente, es un nódulo indoloro en región proximal de extremidades inferiores (Cao E, et al, 2003) aunque otras localizaciones han sido reportadas en la bibliografía consultada como en peritoneo, pared de tórax y abdomen. (Sánchez Wals, 2018; Morales y sus colaboradores, 2009; Montemayor-Sánchez et al, 2015) y otros casos más infrecuentes en mandíbula, (Sarode, 2019) riñón, (Forough, 2019) mama (Martínez-Navarro, 2019) y pulmón. (Miyashita, Ikeda \& Hiraoka, 2019) En su mayoría provienen de la fascia profunda y el músculo esquelético. Su tamaño y la profundidad que alcanza en la dermis, son variables importantes dentro del pronóstico del tumor.

Generalmente esta enfermedad es conocida por su pobre pronóstico con una gran tendencia a metástasis a distancia y un alto índice de recurrencia mayor de un $50 \%$. (Sugihara et al, 2010).

El sarcoma de partes blandas es una proliferación neoplásica mesenquimal extraesquelética y no epitelial, cuyo origen incluye músculo, tendones, tejido fibroso, grasa, vasos, nervios y tejido sinovial. A pesar que estos tumores se reportan con frecuencia representan solo el $0,8 \%$ del total de los que acontecen en el adulto, y causan el 2\% de la mortalidad. (Cao E, 2003) Son procesos poco habituales y su importancia reside en su diagnóstico anatomopatológico precoz, para poder realizar un tratamiento quirúrgico completo. En nuestro Hospital en un período de 20 años solo se ha diagnosticado otro caso, localizado en pared toráxica que ingresó con el diagnóstico presuntivo de un derrame de Moré-Lavallí e inmediatamente evolucionó hacia una tumoración ulcerada de grandes proporciones, falleciendo al mes. 
En los últimos años, se han producido avances notables en el conocimiento de esta enfermedad, pero debido principalmente a la rareza y complejidad de la misma, los resultados no son siempre los óptimos. Dado el peculiar comportamiento de este tumor maligno, es que decidimos describir un caso que presentó una tumoración en la región lateroizquierda del abdomen.

\section{Presentación de caso}

Paciente masculino de 77 años de edad, exfumador desde hace 17 años, que hace aproximadamente 1 año comienza con aumento de tamaño en la región lateral izquierda del abdomen y acude a consulta porque en los 2 últimos meses ha tenido un crecimiento exagerado y sangrado por la superficie de la tumoración.

Al examen físico se constata la presencia de una masa difusa en la región lateral izquierda del abdomen de alrededor de 30x20 cm multilobulada con contornos irregulares y signos inflamatorios donde la tumoración excrecente más grande tiene $20 \mathrm{~cm}$ de diámetro y presencia de áreas de necrosis y zonas sobreinfectadas, con sangrado activo. Adherida a planos profundos que a la palpación refiere solo molestia. Además, al explorar el sistema respiratorio se constata murmullo vesicular disminuido en ambos campos pulmonares.

En los complementarios se precisó anemia con una $\mathrm{Hb} 93 \mathrm{~g} / \mathrm{l}$, Hto $28 \%$ resto de los estudios sanguíneos dentro de parámetros normales. No se observan alteraciones en el ultrasonido abdominal ni en la radiografía de tórax.

En la TAC toracoabdominal se observa adenopatía precarinal de pequeño tamaño, fibrosis apical y en los segmentos posterobasales, bullas subpleurales, presencia de pequeños nódulos de aspecto metastásicos en ambos campos pulmonares. En los cortes de abdomen se observa un proceso ocupativo dependiente de partes blandas en pared lateral izquierda de abdomen, heterogéneo con áreas de necrosis que infiltra cavidad abdominal provocando ligero desplazamiento de asas colónicas, así como ligera distorsión de la grasa perirrenal pero no involucrando las estructuras óseas adyacentes, confirmándose así la tumoración de partes blandas con infiltración abdominal y metástasis pulmonar. Se realiza biopsia por trucut obteniéndose como resultado un sarcoma pleomórfico de origen desconocido.

Por el gran tamaño de la tumoración, la profundidad y la presencia de metástasis se contraindica la cirugía y se indicó quimioterapia con adriamicina e ifosfamida 4 ciclos con intención de detener crecimiento tumoral, la cual no pudo completarse por el fallecimiento del paciente.

\section{Metodologia.}

Se describió un caso recibido por el equipo de cirugía del Hospital Docente de Colón, Matanzas, Cuba Doctor Mario Muñoz Monroy en diciembre del 2019. El diseño de 
investigación se realizó mediante el análisis y la síntesis de la bibliografía lo cual permitió hacer deducciones en relación al desarrollo y proyecciones futuras de casos semejantes.

\section{Resultados.}

Para algunos autores como Forough, 2019; Reshadi, 2014 y Hernández González, 2017 el histiocitoma fibroso maligno es el sarcoma de partes blandas más frecuente en la edad adulta; mientras Li X et al, 2017 reporta la rareza de este tipo de neoplasia. Es posible que esto sea debido a que en la actualidad los criterios para el diagnóstico de esta enfermedad son más restringidos y no se incluyen las formas pleomórficas de otros tumores (rabdomiosarcomas, liposarcomas o fibrosarcomas) (Morales y sus colaboradores, 2009).

El origen de este tumor continúa siendo desconocido hoy día. En un principio se pensó que eran a partir de los histiocitos; en la actualidad la hipótesis histogenética más aceptada es la que sitúa a las células mesenquimales pluripotenciales como precursoras neoplásicas, lo que justifica que en ocasiones coincida temporalmente con los otros tumores antes mencionados. Es un tumor muy agresivo con una tasa de supervivencia a los 5-20 años del 28-33\%. ( Akpinar, 2014)

La presentación clínica del caso objeto de este trabajo como una tumoración indolora con crecimiento rápido y afectación vascular superficial, coincide con lo observado por Hernández González, 2017 en un paciente tratado en Camaguey por un histiocitoma fibroso maligno pleomórfico estoriforme del brazo izquierdo y así lo confirma también Iizumi et al, 2019 en Japón frente a un paciente afectado por esta misma neoplasia en muslo izquierdo. No obstante, existen otras formas menos frecuentes como lo es la masa dolorosa de aparición repentina y síntomas neurológicos o vasculares, relacionados con la localización y origen del tumor.

Pancorbo Sandoval y colaboradores en 2016 presentaron un paciente del sexo masculino de 36 años de edad, que, a partir de un trauma leve, debutó con fiebre, dolor y aumento de temperatura a nivel del muslo derecho, se valoró como un posible hematoma abscedado por lo que requirió ingreso y tratamiento quirúrgico. Posterior al alta y dos meses de evolución, regresa al centro hospitalario por presentar una evolución tórpida con un aumento de volumen de tipo tumoral se ingresó, estudió y se valoró por un equipo de especialistas multidisciplinarios decidiendo una nueva intervención quirúrgica en base a los resultados de laboratorio y estudios imagenológicos, se le realizó exéresis de la tumoración (a nivel del vasto externo) con margen oncológico y enviado para anatomía patológica, informado como un sarcoma pleomórfico.

Los pacientes suelen referir la aparición en un breve periodo de tiempo (que oscila entre varias semanas y algunos meses) de una tumefacción o masa y no es infrecuente que describan un traumatismo previo en la zona afectada. Otros síntomas, como la fatiga y la 
pérdida de peso, no son habituales, pero pueden aparecer en pacientes con enfermedad avanzada. Los tumores de localización retroperitoneal pueden alcanzar un tamaño muy grande antes de ser detectados y los pacientes no sienten una masa como tal, sino síntomas generales asociados al tumor, como anorexia o aumento de la presión abdominal. (Morales y sus colaboradores, 2009).

Una forma inusual de presentación, incluye episodios de hipoglucemia e hiperinsulinemia, fiebre y leucocitosis, los cuales remiten al extirpar el tumor. Un estudio realizado por Hamada et al, citado por Morales y sus colaboradores, 2009 ¡Error! Marcador no definido. relacionó síntomas acompañantes de la tumoración con un aumento en la producción de citokinas (IL-6 especialmente), las cuales producían sintomatología típica de síndrome general (fiebre, pérdida de peso y astenia). Sin embargo, los mecanismos de inducción y el que unos tumores sean productores y otros no, no se han podido aclarar aún.

A pesar de que este tumor puede aparecer en cualquier parte de la anatomía, las tres cuartas partes se localizan en las extremidades. La localización en pared toráxica y abdominal es infrecuente, Sánchez Wals et al, 2018 reportaron un caso operado anteriormente como un lipoma en abdomen, no se indicó biopsia y al mes recidivó; consiguiendo resecar toda la tumoración; mientras en España y en México han publicado un Histiocitoma fibroso maligno pleomórfico cutáneo de rápido crecimiento en el tórax, Morales y sus colaboradores, 2009 y otro en la pared de la región inguinal Montemayor-Sánchez et al, 2015 respectivamente. El caso presentado la tumoración se localizó en la región lateral izquierda de la pared abdominal

Es elemental que los cirujanos estén alertas frente a este tipo de sarcoma, y desde el punto de vista clínico considerar determinados signos de alarma, los cuales fueron propuestos por el Grupo Español de Investigación de Sarcomas (GEIS), constituido por un grupo multidisciplinario de expertos en el estudio y el tratamiento de los sarcomas. (García del Muro, 2011)

Deberán considerarse potencialmente malignas las tumoraciones que afecten partes blandas con cualquiera de los signos siguientes:

1. Tumoraciones mayores de $5 \mathrm{~cm}$.

2. Tumoraciones que hayan experimentado un crecimiento reciente.

3. Tumoraciones profundas (fijas).

De acuerdo a su histología se clasifican en cinco tipos: estoriforme o en "rueda de carreta", mixoide, de células gigantes, inflamatorio y angiomatoide. La variante estoriforme constituye la que más se diagnostica, seguida de la mixoide. Estos subtipos se determinan por técnicas de inmunohistoquímica con marcadores tumorales específicos. (Chintaman, 2011) Aunque los sarcomas pleomórficos son raros, el HFM estoriforme-pleomórfico es el subtipo más frecuente de entre ellos. (Martín, 1998) 
Debido a la ausencia de signos radiológicos característicos, se puede confundir con otras neoplasias como el tumor de células gigantes y otros sarcomas de partes blandas (SPB), por lo que la confirmación histológica es mandatoria.

El diagnóstico de certeza se realiza mediante la histopatología, donde aparecen un pleomorfismo nuclear y citoplasmático, apariencia bizarra de las células estromales y un modelo de crecimiento estoriforme o en remolino, elementos que estuvieron presentes en las muestras obtenidas. La inmunohistoquímica es crucial en el diagnóstico pues permite determinar la variante histológica para establecer la estrategia terapéutica adecuada. (Forough, 2019)

Al paciente se le practicó la biopsia por trucut, que representa un método sencillo, con el cual se puede obtener material suficiente para llegar al diagnóstico histopatológico.

En los estudios imagenológicos de este paciente la radiografía simple y el ultrasonido abdominal no brindan ningún aspecto característico. En ocasiones, los estudios radiológicos muestran afectación de huesos próximos por invasión secundaria, con la posibilidad de fracturas espontáneas. Mientras que en la tomografía se evidencia la presencia de metástasis a abdomen y pulmones.

Resulta importante resaltar lo que plantean Montemayor-Sánchez et al, 2015; para ellos la mayor parte de los sarcomas se diseminan a través de la vía hematógena. La inmensa mayoría de los casos de diseminación metastásica en los sarcomas (incluyendo el FHM) se presenta como enfermedad pulmonar (82\%). Las metástasis en localizaciones extrapulmonares son infrecuentes, pudiendo ocurrir en los ganglios linfáticos (32\%), hueso (15\%) e hígado (15\%). El HFM es un sarcoma maligno con una gran capacidad de recurrencia local (44\%) y de metastatizar (42\%).

El HFM se ha relacionado con múltiples tumores sincrónicos. Según un estudio realizado por Weiss et al, 2007, un 30\% de los 200 pacientes con HFM de su serie, tenían una neoplasia secundaria, no obstante, la presencia de metástasis al momento del diagnóstico no es habitual. Un dato de gran interés, es la asociación de este tumor con enfermedades hematopoyéticas: linfoma de Hodgkin's, linfoma no Hodgkin, mieloma múltiple e histiocitosis maligna. (Montemayor-Sánchez et al, 2015)

Guanghui, 2016 y Umer, 2013 refieren que la edad, la profundidad de la lesión, la variante histológica, la localización, la ausencia de metástasis y el tamaño son los factores pronósticos más importantes. Existen diversos trabajos donde se intentan aclarar los mejores marcadores de malignidad. Para Forough, 2019 y Hernández González, 2017 el factor pronóstico de mayor importancia es el tamaño tumoral, y el grado del tumor primario siendo los tumores de bajo grado aquellos que están formados predominantemente por células pleomórficas estoriformes y las de alto grado los formados predominantemente por células mixoides. 
Bertoni et al citado por Morales y sus colaboradores, 2009 estudiaron 78 casos de HFM localizados en extremidades, observando que los tumores mayores de $5 \mathrm{~cm}$ tenían peor pronóstico y rápida recidiva. Kearney et al, 1980, publicaron un estudio de 167 casos de HFM, para ellos, el factor de peor pronóstico, fue la profundidad que alcanzaba la masa tumoral y la localización distal o proximal. Weiss et al, 2007, indicaron que, a mayor profundidad del tumor, mayor capacidad de recidiva, sobre todo aquellos tumores que afectaban al tejido subcutáneo. También consideran de gran importancia el componente inflamatorio agudo o crónico, postulando, que cuanto mayor es el componente inflamatorio menor es su tendencia a metastizar.

A pesar de las diferentes opiniones de los autores interesados en este tema, parece ser que los mejores marcadores para establecer el pronóstico son, el tamaño tumoral y la profundidad del mismo, además de la localización y el componente inflamatorio del tumor. En nuestro caso el gran tamaño tumoral (mayor de $30 \mathrm{~cm}$ ), asociado a la profundidad del tumor fueron factores de mal pronóstico que finalmente llevaron al fallecimiento del paciente.

El tratamiento de elección es quirúrgico, donde la excéresis total con márgenes oncológicos y la radioterapia adyuvante es la combinación más aceptada para evitar la recidiva local y las metástasis. La quimioterapia no parece incrementar la supervivencia por lo que sólo se reserva para los casos avanzados o que tengan contraindicación para la cirugía (Hernández González, 2017) como ocurrió en este paciente.

El sarcoma pleomórfico indiferenciado es uno de los pocos sarcomas que se pueden presentar como un tumor superficial, es necesario tenerlo siempre en cuenta por parte de dermatólogos, cirujanos, clínicos y médicos de la familia que son los más cercanos a los pacientes pues por su comportamiento agresivo, el mejor pronóstico de curación y sobrevida de los pacientes afectados es la detección precoz y por consiguiente el tratamiento oportuno; de elección el quirúrgico, donde la excéresis total y a tiempo con márgenes oncológicos y la radioterapia adyuvante es la combinación más aceptada para evitar la recidiva local y las metástasis. Esto redundaría en un gran beneficio para los pacientes.

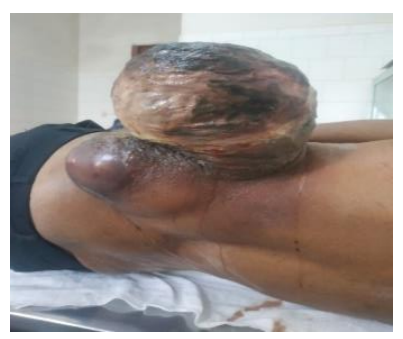

Figura 1 Tumoración de la región lateral izquierda del abdomen

Fuente: Foto tomada al paciente 


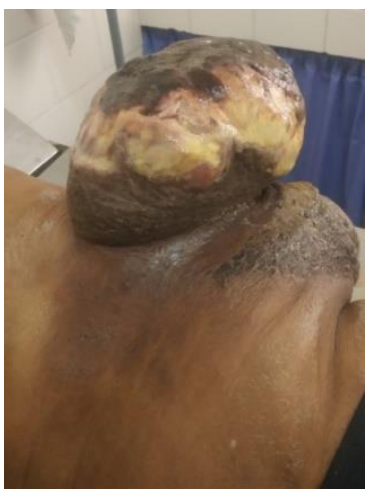

Figura 2 Obsérvese las áreas de ulceración y necrosis

Fuente: Foto tomada al paciente

\section{Conclusiones.}

- El sarcoma pleomórfico indiferenciado es un tumor frecuente de los tejidos blandos, pero en nuestro medio se ha diagnosticado con escasa periodicidad.

- Se pueden presentar como un tumor superficial por lo que es necesario tenerlo siempre en cuenta.

- Debido al comportamiento agresivo de estos tumores, un diagnóstico precoz es vital para lograr un tratamiento correcto lo cual incidiría en una disminución de la morbimortalidad por esta entidad y redundaría en un gran beneficio para los pacientes.

\section{Referencias bibliográficas.}

Akpinar A, Omer CO, Ucler N, Mehmet HI. (2014) Storiform-Pleomorphic Type of Multifocal Malignant Fibrous Histiocytoma of the Lumbar Spine. Am J Case Rep;15(1http://www.ncbi.nlm.nih.gov/pmc/articles/PMC4281027/

Cao E, Martín L, Sánchez J, Del Pozo M. (2003). Sarcoma de la pared abdominal Cir Esp 2003;74(1):43-5 43

Chintamani A. (2011) Soft Tissue Sarcomas-The Pitfalls in Diagnosis and Management. Indian J Surg Oncol;2(4): [about 3 p.]. Disponible en: http://www.ncbi.nlm.nih.gov/pmc/articles/PMC3337196/.

Fletcher CDM, Unni KK, Mertens F. (Eds.) (2002). World Health Organization Classification of Tumours. Pathology and Genetics of Tumours of Soft Tissue and Bone. IARC Press: Lyon 2002.

Forough Ebrahimtabar, Hamid Shafi, Mohammad Ranaee and Mohammad Mehdi Darzi. (2019) A rare case of primary malignant fibrous histiocytoma: a sarcoma of the 
kidney. Ebrahimtabar et al. BMC Urology 19:45 https://doi.org/10.1186/s12894-0190471-7

García del Muro X, Martín J, Maurel J, Cubedo R, Bagué S, De Alavá E, et al. (2011). Guía de prácticas clínicas en los sarcomas de partes blandas. Med Clin;136(9): 408.e1408.e18.

Disponible

en: http://www.sciencedirect.com/science/article/pii/S002577531100176X

Guanghui J, Liu H, Ping Y. (2016) Successful treatment of advanced malignant fibrous histiocytoma of the right forearm with apatinib: a case report. Onco Targets Ther; 9(1) Disponible en: http://www.ncbi.nlm.nih.gov/pmc/articles/PMC4751900/.

Guerra Mesa JL, Lamadrid García J, Rodríguez Machado J. (2017) Histiocitoma fibroso maligno metastásico en estómago. Rev Cub Cir; 56(4). Disponible en: http://revcirugia.sld.cu/index.php/cir/article/view/462.

Hernández González EH; Mosquera Betancourt G; Rondón Martínez E. (2017) Histiocitoma fibroso maligno pleomórfico estoriforme del brazo izquierdo. Rev. Arch Med Camagüey Vol21(3)

Iizumi T, Shimizu S, Numajiri H, Takei H, Yamada N, Mizumoto M, Ishikawa H, Okumura T, Sakurai H. (2019) Large Malignant Fibrous Histiocytoma Treated with Hypofractionated Proton Beam Therapy and Local Hyperthermia. Int J Particle Ther

Kearney MM, Soule EH, Ivins JC. (1980) Malignant fibrous histiocytoma. A retrospective study of 167 cases. Cancer 1980; 45:167-78.

Li X, Liu R, Shi T, et al. (2017) Primary pulmonary malignant fibrous histiocytoma: case report and literature review. J Thorac Dis. 2017;9: E702-E708.

Martín E, Arnau A, Cantó A. (1998) Fibrohistiocitoma maligno de pared torácica. Recidiva temprana tras cirugía. Arch Bronconeumol 1998; 34:467-8.

Martínez-Navarro J, Fumero-Roldan L, Izquierdo-Rey E. (2019). Sarcoma pleomórfico indiferenciado de la glándula mamaria. Revista Electrónica Dr. Zoilo E. Marinello Vidaurreta Vol. 44, número 3 ISSN 1029-3027 | RNPS 1824 mayo-junio 2019.

Miyashita Y, Ikeda N, Hiraoka K. (2019) Primary undifferentiated pleomorphic sarcoma (Malignant fibrous histiocytoma) of the lung: A case report. Clin Case Rep. 2019; 00:1-5. Disponible en: https://doi.org/10.1002/ccr3.2517.

Montemayor-Sánchez D, Robles-Atayde JA, Reynoso Rivera-Río LR, Decanini-Arcaute H. (2015) Manejo quirúrgico de sarcoma pleomórfico de pared abdominal. Reporte de un caso. Cirugía Plástica; 25 (1): 23-28 Disponible en: www.medigraphic.com/cirugiaplastica

Morales AL, Zaballos P, Ros C, Grasa MP, Carapeto FJC. (2009) Histiocitoma fibroso maligno pleomórfico cutáneo de rápido crecimiento. Med Cutan Iber Lat Am 2009; 37(1):58-63. 
O'Brien JE, Stout AP. (1964). Malignant fibrous xanthomas. Cancer; 17:1037-93.

Pancorbo Sandoval EA, Delgado Quiñones A, Díaz Prieto G, Hernández Hernández J, Pinto Contreras LA. (2016) Sarcoma pleomórfico. Presentación de un caso. Rev Méd Electrón; 38(1). Disponible en: http://www.revmedicaelectronica.sld.cu/index.php/rme/article/view/2920/1412

Pathrose G, John NT, Manojkumar R. (2015) A rare case of malignant fibrous histiocytoma/ pleomorphic undifferentiated sarcoma of the kidney. J Clin Diagn Res. 2015; 9(1): Pd27-9. 1.

Reshadi H, Rouhani A, Mohajerzadeh S, Moosa M, Elmi A. (2014). Prevalence of Malignant Soft Tissue Tumors in Extremities: An Epidemiological Study in Syria. Arch Bone Jt Surg;2(2): [about 6p.]. Disponible en: http://www.ncbi.nlm.nih.gov/pmc/articles/PMC4151447/.

Sánchez Wals L, Vázquez González CL, Castellanos Prada A, Marcasciano M, Crespo Álvarez A. (2018) Reconstrucción de la pared abdominal con colgajo miocutáneo transverso de recto abdominal. Revista Cubana de Cirugía. 2018;57(1):72-77 Disponible en: http://scielo.sld.cu

Sarode SC, Sarode GS, Ingale Yb, Ingale M, Raj AT, Patil S. (2019) Malignant fibrous histiocytoma of the mandible - A case report and review of published case reports. Journal of Oral Biology and Craniofacial Research 9 221-225

Sugihara T, Fujimura T, Kume H, Homma Y. (2010) Successful treatment of metastatic malignant fibrous histiocytoma of the kidney. Urol Int. 2010;85(1): 118-20.

Umer HM, Umer M, Qadir I, Abbasi N, Masood N. (2013) Impact of Unplanned Excision on Prognosis of Patients with Extremity Soft Tissue Sarcoma.Sarcoma. Disponible en: http://www.ncbi.nlm.nih.gov/pmc/articles/PMC3659434/

Weiss S, Goldblum J. (2007) Enzinger and Weiss's soft tissue tumor, ch 14. Philadelphia, PA: Mosby Elsevier;403-428.

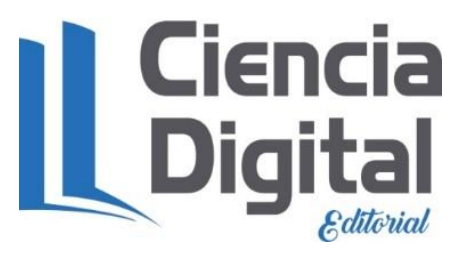


PARA CITAR EL ARTÍCULO INDEXADO.

Lantigua Hernández, O., González Díaz, Y., Morales Wong, M. M., \& Alfaro Alonso, R. (2021). Sarcoma Pleomórfico Indiferenciado. A propósito de un caso . Anatomía Digital, 4(1), 101-112. https://doi.org/10.33262/anatomiadigital.v4i1.1533

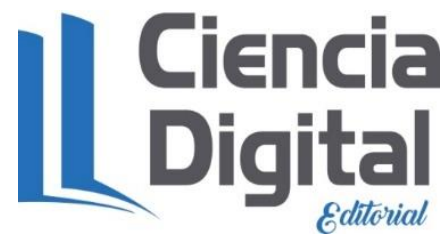

El artículo que se publica es de exclusiva responsabilidad de los autores y no necesariamente reflejan el pensamiento de la Revista Anatomía Digital.

El artículo queda en propiedad de la revista y, por tanto, su publicación parcial y/o total en otro medio tiene que ser autorizado por el director de la Revista Anatomía Digital.
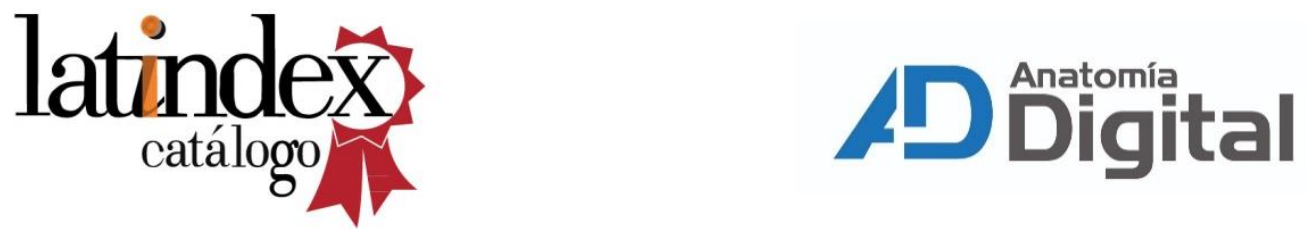